\title{
Optimal road design through ecologically sensitive areas considering animal migration dynamics
}

\author{
Nicholas Davey $^{\mathrm{a}, *}$, Simon Dunstall ${ }^{\mathrm{b}}$, Saman Halgamuge ${ }^{\mathrm{c}}$ \\ ${ }^{a}$ The University of Melbourne, Parkville, Australia \\ ${ }^{\mathrm{b}}$ CSIRO Data 61, Docklands, Australia \\ ${ }^{\mathrm{c}}$ The Australian National University, Canberra, Australia
}

\section{A R T I C L E I N F O}

\section{Article history:}

Received 5 July 2016

Received in revised form 17 February 2017

Accepted 18 February 2017

Available online 24 February 2017

\section{Keywords:}

Road design

Ecological constraints

Surrogate model

Dimensionality reduction

Spatially-explicit model

Genetic algorithm

\begin{abstract}
A B S T R A C T
With increasing land transportation requirements in both urban and rural areas, roads are encroaching ever more on animal habitats, where collisions with vehicles are a leading contributor to wildlife mortality. While road designers recognise the importance of accounting for such impacts at the design level, existing approaches simply either ignore viable habitat or avoid such regions entirely. Respectively, this can result in road alignments that are overly damaging to vulnerable species or prohibitively expensive to build and operate. The research presented in this paper investigates the effects of explicitly accounting for animal mortality on the design of a road through an ecologically sensitive area. The model presented achieves this by incorporating a spatially-explicit animal migration and road mortality model into an accepted optimal road alignment algorithm to propose low-cost roads that maintain the animal population above a minimum threshold by the end of a specified design horizon. The new method was applied to an example scenario to demonstrate the effect of setting a minimum required animal population on the road design. This model was able to consistently produce a road that met a minimum required species conservation benefit. This reflected a major improvement over the model that ignored animal habitats while only requiring a minor increase in construction and operating costs compared to the model that avoids habitat.
\end{abstract}

(c) 2017 Elsevier Ltd. All rights reserved.

\section{Introduction}

Roads are an integral part of modern transportation networks and much work has been done to develop algorithms that produce low-cost road alignments through real terrain that incorporate many realistic cost features and constraints (Kang et al., 2012). However, roads also affect surrounding wildlife populations (Forman and Alexander, 1998; Friedrich, 2015) and in some cases, these impacts are the deciding factors in selecting the final road design (Kang et al., 2009). Therefore, it is crucial to properly account for these ecological effects during the road alignment optimisation process.

Similarly, while ecological models can account for many of the negative impacts of roads, they currently only focus on existing roads or general policy recommendations. As animal populations can be highly sensitive to the actual route taken by a road, it is imperative that these ecological models be incorporated during the calculation of a new road's path. This will

\footnotetext{
* Corresponding author.

E-mail addresses: ndavey@student.unimelb.edu.au (N. Davey), Simon.Dunstall@data61.csiro.au (S. Dunstall), saman.halgamuge@anu.edu.au (S. Halgamuge).
} 
allow road designers to determine optimal alignments while also maintaining the long term ecological sustainability of new roads.

Optimal road design through a three dimensional terrain consists of two principal components: horizontal alignment, which is the road path when viewed from above; and vertical alignment, which is the road profile. The overall objective is to minimise the expected present value of road construction costs and lifetime operating costs subject to numerous constraints. Many different approaches have been considered in the literature for road design to date: variational calculus, network optimisation, dynamic programming, enumeration, linear programming, numerical search, distance transformation, neighbourhood search heuristics, genetic algorithms (Kang et al., 2012) and derivative-free approaches (Mondal et al., 2015). Of these, seven have successfully been applied to simultaneously solving horizontal and vertical alignments: numerical search using variational calculus (Chew et al., 1989), distance transforms (De Smith, 2006; Li et al., 2016), neighbourhood search heuristics (Aruga, 2005; Shafahi and Bagherian, 2013), genetic algorithms (GA) (Jha et al., 2006; Jong and Schonfeld, 2003; Kang et al., 2012; Kim et al., 2005; Maji and Jha, 2012; Mishra et al., 2014), dynamic programming (Li et al., 2013) and derivative-free methods (Mondal et al., 2015). Of these approaches, evolutionary-based techniques such as genetic algorithms have been the most widely-investigated due to their ability to realistically handle the complex fitness functions featuring non-linearities and discontinuities while including mechanisms to escape local optima.

Despite their success, some important limitations of GAs must be mentioned. The first is that they do not guarantee optimality (including local optimality). They simply allow finding less-expensive road profiles. Furthermore, the stochastic nature of the algorithm means that the same solution may not be achieved each time the algorithm is run. Additionally, a very large number of evaluations are required to sufficiently explore the solution space, which can cause tractability problems, particularly if the evaluation function is computationally expensive (Jin, 2011). There are black-box optimisation tools such as NOMAD (Abramson et al., 2016; Audet et al., 2009) and Hopspack (Plantenga, 2009) that implement derivative-free approaches (Audet and Dennis, 2006) to overcome some of these issues. Such tools are fast and guarantee at least local optimality. This has been successfully applied in the work of Mondal et al. (2015) in which the authors develop a very efficient and robust bi-level optimal road design model in a specified corridor where optimal earthwork costs are computed using Mixed Integer Linear Programming (Hare et al., 2014). Finally, in another recent paper, Li et al. (2016) extend the distance transform approach of De Smith (2006) to consider highly-constrained railway alignments in mountainous regions using adaptive neighbouring masks and a bi-directional scanning strategy. This method addresses the difficulty of finding suitable alignments through complex mountainous terrain.

In addition, recent literature into optimal road design also recognises the increasing importance of adequately accounting for socio-economic and environmental effects of these designs (Kang et al., 2012; Mishra et al., 2014). For instance, in industries such as mining, where new roads are regularly built, regulators and communities at large could potentially shut down operations if they breach social and environmental constraints (Cruz and Wakolbinger, 2008; Prno and Slocombe, 2012). On public roads, the damaging effects on wildlife are also becoming increasingly dominant. For example, in the United States, it is estimated that anywhere between 15 and 20\% of land mass is already ecologically impacted by roads (Forman and Alexander, 1998) with vulnerable species being affected through mechanisms such as habitat fragmentation and degradation, modification of animal behaviour and vehicle collisions (Dique et al., 2003b; Jaeger et al., 2005).

While road design algorithms can be modified to apply penalties to roads traversing animal habitats (Kang et al., 2012: Jong and Schonfeld, 2003; Chew et al., 1989; Kim et al., 2005; Maji and Jha, 2012), current approaches are too simplistic. In these cases, an arbitrary penalty cost is applied to sensitive regions of the map. This approach unfortunately ignores the dynamics of animal behaviour, which depends on the overall road path and its effects on highly mobile species. This has the potential to produce designs that mask these important dynamics, thereby creating roads that may either be too expensive to construct and operate or too damaging to nearby animal populations.

Fortunately, empirical studies have measured ecological impacts of roads. For example, Fahrig and Rytwinski (2009) conducted a large survey on quantifiable measures of the impacts of existing roads on a number of animal species due to various mechanisms. These include noise, pollution and habitat fragmentation. In another study, Jaarsma (1997) investigated the effects of "traffic-calmed rural areas" in reducing contact with animal species. They found that the ability to route traffic along high capacity distributor roads to accommodate increasing traffic volumes results in greater species survival in the long run.

Empirical studies based on real-world data such as these have formed the basis for models to evaluate proposed road designs. For instance, van Langevelde and Jaarsma (2009) extended the work of Jaarsma (1997) to investigate the effects of different traffic policies within an existing network on species survival when traffic volume is varied. Focusing instead on fixed traffic volumes, Frair et al. (2008) assessed different road alternatives in the vicinity of habitat zones by varying road density. In another study, Rhodes et al. (2014) used a comprehensive animal movement and mortality model from Rhodes et al. (2005) to show scenarios under which it is suitable to build many smaller roads or fewer large ones. This model used specific species data relating to relative habitat preference, foraging and home ranging behaviour and the location of vegetation throughout the region to develop realistic transition probabilities for animals in the region. These transitions have corresponding survival probabilities that are related to the traffic volume and the number of road crossings required.

The model of Rhodes et al. (2014) was also used in Davey et al. (in press) to observe the effect of optimally controlling traffic flow over time to maintain a species population above a critical threshold while maximising the present value of the road. This was used to select the most valuable road to build among a fixed set of candidate roads. 
Models capturing the spatial dynamics of animals can also be used where wildlife crossings are installed to mitigate the hazard of crossing roads. This is important because wildlife crossings and fencing have been effectively employed in many locations around the world to improve conservation (Alexander and Waters, 2000). However, such mitigation measures can often be extremely expensive. To address this, Polak et al. (2014) used the model from Rhodes et al. (2014) to evaluate the benefit of different configurations of protective fences and wildlife bridges on species survival for an existing small road network. In their work, they analyse the trade-off between mitigation cost and benefit in a koala-inhabited region in Southern Queensland. This raises further research opportunities to determine optimal bridge and fence locations using computational models, which in practice are typically determined by tracking actual animal movements over time (Alexander, 2008).

Importantly, incorporating a spatially-explicit animal migration and mortality model into the road optimisation process will allow road designers to set critical population thresholds required for species survival. Grimm and Wissel (2004) showed through mathematical modelling that for any given time in the future, a species' survival probability dramatically increases once a safe population threshold is met. This threshold allows the species to survive in the face of stochastic factors such as disease, climate change, predation, genetic diversity (Frankham, 1995, 1996) and other catastrophes. Furthermore, this threshold is inversely related to the carrying capacity of the region and to the underlying population growth rate (Grimm and Wissel, 2004; Lande, 1993; Fahrig, 2001).

In Section 2 of this paper, we describe our approach that incorporates an animal movement and mortality model into a road design algorithm to find the optimal alignment through an ecologically sensitive region. Starting with a genetic algorithm (GA)-based road design model (Jong and Schonfeld, 2003), we incorporate an empirically-tested ecological movement and mortality model (Rhodes et al., 2014) within the road fitness evaluation to guide the optimisation process to ensure that the road produced satisfies a minimum conservation benefit. The conservation benefit is defined as the ratio of the expected species population at the end of the design horizon when the road is built to when no road is built at all (Polak et al., 2014). The incorporation of the two models is achieved using a surrogate function and a novel ecological parameter that together improve computational tractability of the problem.

The model is applied to a numerical example in Section 3 to compare its performance against the existing approaches. We also demonstrate how the ecological movement model can be used to develop a visual representation of the impact caused by building the road through specific locations within the map. This visual representation can assist road designers to better understand the relative sensitivity of the animal population to building the road through different locations within the map. Finally Section 4 investigates the effect of varying the required conservation benefit and traffic flow rate for the example region considered. In all cases, the algorithm presented in this paper is able to find a less expensive road while satisfying a required end population of animals.

\section{Algorithmic approach}

The road design algorithm proposed in this paper consists of two integrated models: 1 . an overall road design algorithm based on Jong and Schonfeld (2003) using a genetic algorithm, and 2. an animal movement and mortality model based on Rhodes et al. (2014), which is incorporated into the fitness function of model 1. As evaluation of the final population can be computationally intensive, a surrogate model is employed to estimate the end population for each road iteration. This surrogate model is updated over time by computing the full model of Rhodes et al. (2014) for a subset of roads at each generation to update the functional relationship between the input parameters and the estimated end population. In addition, the animal movement and mortality model is also used to determine the areas of the region through which it is more damaging to build a road. The resulting map provides a visual representation of the damage incurred by building the road through certain locations.

Similar to existing research (Polak et al., 2014; Rhodes et al., 2014; van Langevelde et al., 2009), the model presented in this paper considers a single species in an ecologically sensitive area. This species could be of significant economic and cultural value to the area (Dique et al., 2003b) or particularly vulnerable to vehicle collisions (Fahrig and Rytwinski, 2009). That said, the model can also be extended to multiple species and sub-regions by separately considering animal migration and mortality and required thresholds for each individual species or sub-region. When dealing with multiple species, the users may also need to account for ecosystem features related to the species' co-existence in the same region. This includes potential predation and symbiosis (Fahrig, 2003; Menge and Sutherland, 1976). If significant, these features will affect the embedded simulation model used in the road design. To simplify the analysis, we assume that the quality of habitat patches, the age structure of the animal population and the traffic volume remain constant throughout the design horizon. In addition, other ecological effects of roads such as noise, pollution and inhibition of gene flow (Holderegger and Di Giulio, 2010) are ignored. Finally, we do not consider constructing fences or wildlife crossings. Although they would be interesting extensions to the model, they are outside of the scope of this paper.

\subsection{The road design model}

The genetic algorithm (GA) approach of Jong and Schonfeld (2003) is used to model the geometric road design. This algorithm explores the solution space of possible roads to find a better road alignment through a complex three-dimensional terrain such as that shown in Fig. 1. As the solution space can be highly non-linear, discontinuous and may contain multiple 


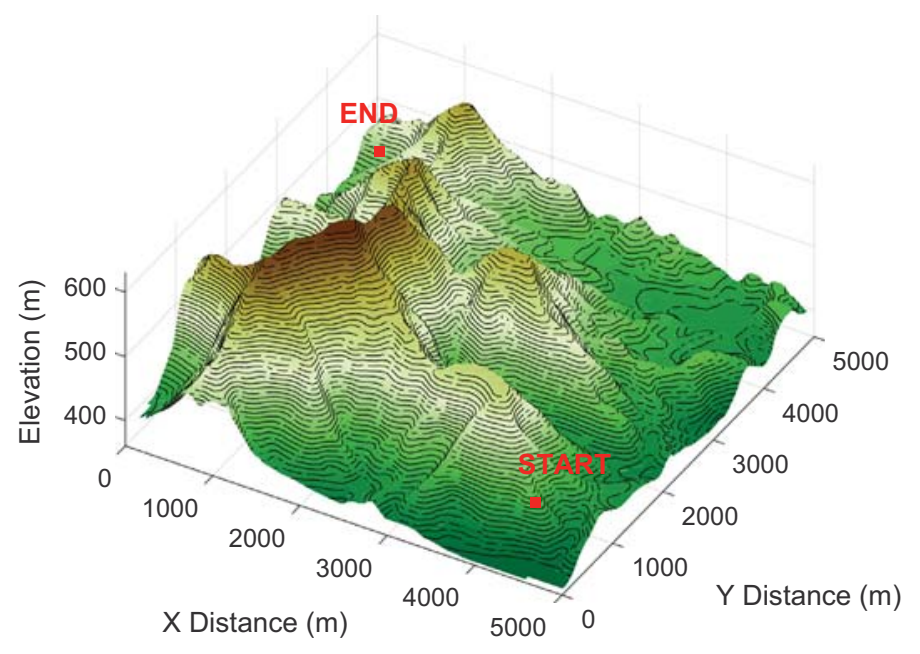

Fig. 1. Example of realistic terrain.

local optima, population-based algorithms such as GAs are suitable (Kang et al., 2012). We also deemed the GA approach appropriate for incorporating a spatially-explicit animal movement model as it can take an arbitrary cost function. This is important because the animal movement and mortality model described in Section 2.2 requires complete road paths in order to evaluate animal impact. The requirement of evaluating complete roads rules out methods such as distance transforms, which do not evaluate a population of candidate roads. Finally, as the main aim of the paper is the incorporation of an ecological model within existing road design approaches and not on the specific road design algorithm used, we have chosen a well-established and easy-to-implement road design algorithm. Although, it should be noted that recent advances in derivative-free and black-box algorithms may provide compelling alternatives that should improve computation times (Mondal et al., 2015).

The GA road optimisation framework consists of two major components: 1. road design and evaluation and 2. the genetic algorithm used to find the best road.

In design and evaluation, an individual road is uniquely described by $N$ design points $\left(P_{i}=\left[x_{i}, y_{i}, z_{i}, r_{i}\right]\right)$ where $x_{i}, y_{i}$ and $z_{i}$ are the coordinates of point $i$ in three-dimensional space and $r_{i}$ is the corresponding radius of curvature. The radius of curvature is determined from the required design speed, maximum superelevation angle and other geometric considerations as stipulated in AASHTO (2011). These points represent the points of intersection of connected straight line segments joining a start point $\left(P_{0}\right)$ to an end point $\left(P_{N+1}\right)$, where $N$ is the number of intervening intersection points between the start and end points. This is used with input data obtained from a geospatial information system (GIS) database to compute and evaluate the road design. The intersection points are encoded as a vector of real numbers, which fully defines an individual in the genetic algorithm. The intersection points are first used to generate smooth horizontal and vertical alignments. These alignments are used to compute the road's costs according to design standards outlined in AASHTO (2011) in order to satisfy safe travel speeds, stopping sight distances, earthwork requirements, grade requirements and to avoid forbidden regions. Further details for implementing this design can be found in Jha et al. (2006), Jong et al. (2000), and Jong and Schonfeld (2003), with more advanced additions in Casal et al. (2017) and Kang et al. (2012).

Once each road alignment has been designed, its overall cost is evaluated using a comprehensive fitness function. This cost consists of five main components: earthwork costs, location costs (right-of-way, soil stabilisation), length-dependent costs (fuel, pavement, travel time, environmental), accident costs and habitat-degradation costs (Eq. (1)). The first four categories of costs have been developed in Kang et al. (2012), Jha et al. (2006) and Jong and Schonfeld (1999). The minimum animal population constraint is included in the objective function by explicitly evaluating the expected population of animals at the end of the design horizon $\left(K_{T}\right)$. If this end population is below the minimum required population level $\left(K_{\min }\right)$ for ecological sustainability (Grimm and Wissel, 2004), then a large penalty is added to the overall cost of the road, as shown in Eq. (2). In this equation $1\left(K_{T}<K_{\min }\right)$ is equal to 1 for $K_{T}<K_{\min }$ and 0 otherwise. The method for computing the end population using Monte Carlo simulation and a spatially-explicit animal movement and mortality model is described in detail in Section 2.2 .

In a practical situation, the designer would need to choose an appropriately-sized penalty cost per animal below the required end population threshold $\left(C_{\text {per_animal }}\right)$ based upon the specific application. If meeting this threshold is crucial, the designer would set the penalty cost to be appropriately large in comparison to the other road costs. One approach to determine this scaling would be to run the model ignoring animal habitats first to ascertain a low-value road and then set the penalty cost commensurate with this.

$$
C_{\text {road }}=C_{\text {earthwork }}+C_{\text {Location }}+C_{\text {Length }}+C_{\text {Accident }}+C_{\text {Penalty }}
$$




$$
C_{\text {Penalty }}=C_{\text {per_animal }}\left(K_{\min }-K_{T}\right) 1\left(K_{T}<K_{\min }\right)
$$

The genetic algorithm also takes advantage of five unique population creation functions to ensure a high genetic diversity at the initial time step as well as eight problem-specific crossover and mutation operators as outlined in Jong and Schonfeld (2003). These collectively ensure that the algorithm adequately explores the solution space, accounting for the advantages of straight roads, while effectively introducing curves around obstacles where needed (Kang et al., 2009).

\subsection{The animal movement and estimated mortality model}

Unlike existing models, the cost function used in this paper does not penalise roads passing through infeasible areas by simply applying a large right-of-way cost proportional to the amount of habitat crossed. Instead, it uses a spatially-explicit model (Rhodes et al., 2014) that accounts for the movement and mortality of the animal species over the design life by taking into account animal locations and ecological characteristics. This model explicitly determines the conservation benefit $(\rho)$ of each road by computing the end populations of the species in each habitat patch based on actual population dynamics. The conservation benefit $(\rho)$ is defined as the expected size of the animal population in the entire region at the end of the design horizon relative to the expected end population given that no road is present (Eq. (3)) (Polak et al., 2014). In this equation, $\kappa_{k}(T)$ is the population in patch $k$ at time $T$.

$$
\rho=\frac{\mathbb{E}\left[\sum_{k} \kappa_{k}(T)\right]}{\mathbb{E}\left[\sum_{k} \kappa_{k}(T) \mid \text { no road }\right]}
$$

To achieve this, geospatial data is introduced in a raster grid format. The use of such data is common (Lunney et al., 1998) and widely available on public GIS databases held with government authorities such as State of Queensland Department of Natural Resources and Mines (2016). As the resolution of this data is sometimes too great to allow a tractable problem, a coarser grid can be computed as shown in Fig. 2 by aggregating nearby cells. In this aggregation, the patches of the model are contiguous areas of the same habitat type that are not split by grid lines of the coarse grid or the road. Although not treated as patches in the model, non-habitat regions may still be crossed by animals if the distance is small enough. Once all of these habitat patches $(k \in[1, \ldots, m])$ are identified, their areas $\left(A_{k}\right)$, centroids $\left(\overline{\mathbf{c}}_{k}\right)$ and initial populations $\left(\kappa_{k}\right)$ are computed. For computational purposes, it is assumed that on average all animals reside at the centroids of their current patches (Rhodes et al., 2014).

It should be noted that larger study regions will require more patches. This is because the sizes of the patches must not be too large otherwise the model will not be able to capture the smaller, more likely migration distances. However, as the movement and mortality model requires computing the transition from each patch to every other patch, the computation time increases with the square of the number of patches. Furthermore, some studies may warrant sub-regional considerations and constraints. These factors could be incorporated by treating each sub-region as containing a separate species with unique characteristics and/or setting unique minimum population thresholds for each region.

The animal movement model is then used to simulate the population in each of these patches over time in the presence of traffic along the road using transition and survival probabilities (Polak et al., 2014; Rhodes et al., 2005, 2014). The transition probabilities of migrating from one patch to another $\left(T P_{a \rightarrow b}\right)$ are computed using the model outlined in Rhodes et al. (2014). This model computes the probability of an animal reaching a new location based on the distance of the originating patch to the destination patch $\left(r_{a b}=\left|\overline{\mathbf{c}}_{a}-\overline{\mathbf{c}}_{b}\right|\right)$, the relative preference of the destination habitat to primary/secondary habitat $\left(\alpha_{j}\right)$, the

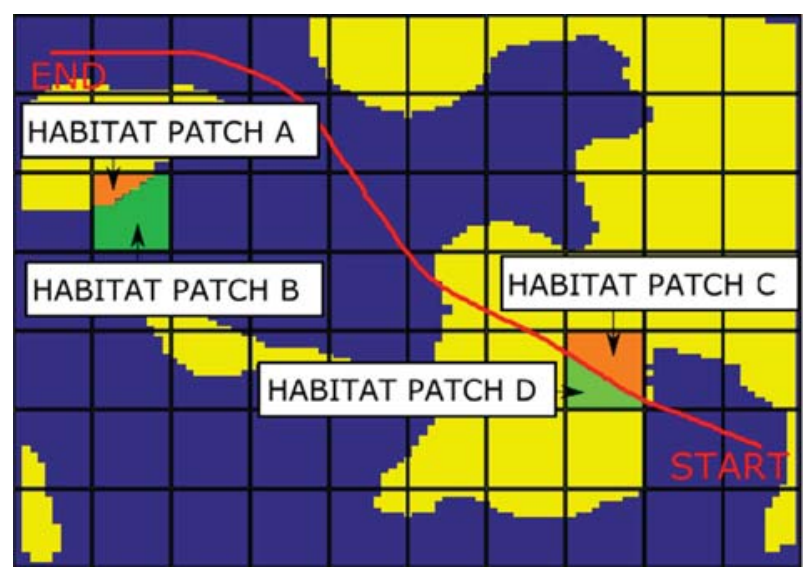

Fig. 2. Splitting a grid with a road present into discrete habitat patches. Example patches A and B are created by splitting a grid cell into distinct habitat types. Example patches $\mathrm{C}$ and $\mathrm{D}$ are created by splitting a grid cell along the road boundary. 
distance of the destination patch to the centre of the animal's home range $\left(r_{b h}\right)$, normalised by the relative preference of all patches in the grid. The home range is defined as the location where the animal spends most of its time Rhodes et al. (2005).

Using this model, for every originating patch, the probability of migrating to a destination patch is determined by Eqs. (4)-(6) (Rhodes et al., 2014). Here, $j \in[1, \ldots, n]$ is the habitat type, $k \in[1, \ldots, m]$ represents the patches, $1(x, y)$ is the indicator function of whether patch $x$ is of habitat type $y$, and $w_{j}$ and $\phi_{a b}$ are given by Eqs. (5) and (6), respectively.

$$
\begin{aligned}
& T_{a \rightarrow b}=\frac{\phi_{a b} \sum_{j=1}^{n}\left[w_{j}(b) \mathbb{1}(b, j)\right]}{\sum_{k=1}^{m}\left\{\phi_{a k} \sum_{j=1}^{n}\left[w_{j}(k) 1(k, j)\right]\right\}} \\
& \phi_{a b}=\frac{\lambda e^{-\lambda r_{a b}}}{2 \pi r_{a b}} \\
& w_{j}(b)=e^{\alpha_{j}+\beta r_{b h}}
\end{aligned}
$$

In Eq. (4), $\phi_{a b}$ is the habitat-independent movement probability function (Rhodes et al., 2005), which defines the movement probability in the absence of species habitat preferences. $\lambda$ is the negative exponential scale parameter, where smaller values indicate a higher tendency for the species to travel larger distances in a fixed time. Additionally, $w_{j}$ is the habitat preference parameter, where $e^{\alpha_{j}}$ is the relative preference for habitat $j$ compared to primary/secondary habitat and $\beta$ represents the attraction of an individual to its home range. Larger negative values of $\beta$ indicate a stronger attraction between the animal and its home range. Finally, $r_{b h}$ is the distance between the destination patch and the animal's home range. These parameters can be obtained for different animal species using the approach described in Rhodes et al. (2005). In this approach, movement pairs (i.e. single movements from one location to another) over a region with a known vegetation map are used. The model parameters are then estimated as those that minimise the negative log-likelihood of the data.

Computing this formula for all transitions results in a square matrix (TP) of the probability of transition from each patch to every other patch. The rows of this matrix represent the originating patches and the columns, the destinations. As the matrix deals with probabilities, each row sums to 1 .

Similarly, the probability of survival $\left(S P_{a \rightarrow b}(t)\right)$ of moving from patch $a$ to patch $b$ at time $t$ is defined by Eq. (7). Here, $Q$ is the two-way traffic volume at time period $t, W_{V}$ is the average vehicle width, $l$ is the average animal head-to-tail length, $v$ is the average animal crossing speed and $g_{t} r$ is the annual traffic growth rate. We assume that an animal crosses perpendicular to the road at constant velocity. Furthermore, we have limited our analysis in this paper to only consider flow rates instead of traffic speeds or measures to improve animal visibility. In fact, various authors (Dique et al., 2003b; Friedrich, 2015; Polak et al., 2014) have observed that although varying traffic speeds and improving visibility of animals may mitigate the effects of roads, studies have had mixed results. Friedrich (2015) and Jaeger et al. (2005) also point out that traffic volumes are the dominant factor in animal collisions with vehicles.

$$
S P_{a \rightarrow b}(t)=e^{-Q \frac{W_{V}+l}{v}\left(1+g_{t} r\right)^{t}}
$$

The population in each patch at the end of the current time period $\left(\boldsymbol{\kappa}_{e}(t)\right)$ is updated to account for this movement and mortality by multiplying TP and SP using Eq. (8). Here, $\odot$ denotes element-wise multiplication and $\boldsymbol{\kappa}_{s}(t)$ denotes the starting population in each patch as a column vector.

$$
\boldsymbol{\kappa}_{e}(t)=[\mathbf{T P} \odot \mathbf{S P}] \boldsymbol{\kappa}_{s}(t)
$$

As the life-cycle for a road is in the order of decades or more, a population growth/reduction model based on logistic growth is used. Eq. (9) computes the population multiplier $\left(\zeta_{k}\right)$ for each population patch to account for births and deaths (excluding road mortality). Here, $g_{p} \sim N\left(g_{p \mu}, g_{p \sigma}\right)$ is the population growth rate parameter and $\left(\eta_{j}\right)$ is the capacity per square metre for habitat type $j$.

$$
\zeta_{k}=1+g_{p} \frac{\sum_{j=1}^{n} \eta_{j} \rrbracket(k, j)-\kappa_{k}}{\sum_{j=1}^{n} \eta_{j} \mathbb{1}(k, j)}
$$

Finally, Eq. (10) computes the population in each patch at the start of the next time period, where $\zeta$ is a column vector.

$$
\boldsymbol{\kappa}_{s}(t+1)=\boldsymbol{\kappa}_{e}(t) \odot \zeta
$$

As the population growth rate is uncertain, Monte Carlo simulation is used to estimate the species population at the end of the design horizon for each road in the optimisation process. To do this, the animal movement and mortality model is used to simulate the changing animal populations throughout the grid from time $t=0$ to $t=T$. At each time step, the population in each patch is first updated using Eq. (8) to compute the animal movements and mortalities based on the patch population densities at time $t$. Next, the growth rate parameter, $g_{p}$, in each habitat patch is sampled from a normal distribution. These growth rates are then used to update the populations of the patches for the next time period using Eq. (9). As the spatial distribution of animals in the region varies over time, the proportion of animals killed due to the road is not a stationary process. This simulation is repeated many times to generate a large number of Monte Carlo paths. The average of the end populations for these paths is the estimate of the final population for the road. 


\subsection{Incorporating animal movement and mortality into road design}

Directly incorporating the full animal movement and mortality model into the road fitness function significantly increases the computational complexity of the road evaluation. As the GA requires the evaluation of potentially thousands of roads, this greatly reduces the tractability of the problem. To overcome this, we propose using a surrogate model $(f)$ to estimate the final animal population to avoid computing the full model for every road. The use of surrogate models is widespread in engineering optimisation problems where the fitness function is computationally intensive (Forrester et al., 2008; Jin, 2005, 2011).

At each generation of the GA, a small number of roads are randomly sampled from the prevailing population of roads. The end animal population for each of these sampled roads is then computed using the full model of Section 2.2. The results of these full model computations, as well as the intersection points defining the roads themselves, are stored in an array of full results $\left(\mathcal{D}_{1}\right)$ that is retained for use in future generations. A regression line is fitted to the results stored in $\mathcal{D}_{1}$ to create the surrogate model. This model is used to estimate the end animal populations for the remaining roads. Finally, crossover and mutation are performed on the current generation of roads in order to produce the next generation in the GA.

\subsubsection{Dimensionality reduction in the surrogate model}

An individual road design features $N$ intersection points in three dimensions, which means that the number of degrees of freedom of the surrogate model is $3 N$. For any useful road design, the number of intersection points will typically be more than 10 (Kang et al., 2009). Therefore, the number of dimensions of the surrogate model will typically be more than 30 . Problems with this many dimensions are known to pose challenges for surrogate models (Gorissen et al., 2010).

To reduce this dimensionality, a novel ecological impact factor is introduced. This factor, called Initial Animals at Risk (IAR) represents the proportion of animals that are expected to perish in the first time period for a single road due to road traffic. It is calculated using Eq. (11).

$$
I A R=1-\frac{\sum_{k} \boldsymbol{\kappa}_{e}(0)}{\sum_{k} \boldsymbol{\kappa}_{s}(0)}
$$

This value is chosen as a proxy for the ecological impact of the road because roads with a higher IAR are expected to have a lower animal population at time $t=T$. The surrogate model is computed by fitting a locally-linear, kernel-based regression (Hastie et al., 2009) to the plot of end population versus IAR for all roads stored in $\mathcal{D}_{1}$. With each subsequent generation in the GA, the road population is expected to approach the globally optimal road. Therefore, the error between the full model calculations and the surrogate model's prediction of end population is expected to diminish.

The overview of the entire road design process, including the computation of IAR and updating of the surrogate model is shown in Fig. 3.

\subsection{Output IAR map}

The surrogate model is useful for producing a reasonable estimate of the end population when running the GA. However, it does not provide spatial information of the animal mortality caused at specific locations of the map if they are occupied by a road. This spatial representation can be useful to road designers and ecologists in understanding what the most ecologically sensitive areas of the region are. Although habitat and vegetation maps identify which locations of a study region contain viable animal habitat, we cannot necessarily expect that all locations with the same habitat type will be impacted equally if a road is built through them due to species dynamics.

Similar to the approach presented in Section 2.3.1, we use the animal movement and mortality model of Rhodes et al. (2014). The model allows us to develop a map that shows the contribution to IAR caused by building a road in each location of the terrain. By determining the individual grid cells traversed by a road, the overall IAR can be estimated by adding up the IAR of each of these cells using Eq. (12).

$$
I A R_{\text {road }} \approx \sum_{\text {cell }} I A R_{\text {cell }}
$$

We consider the initial terrain as a region containing $M$ discrete grid cells. To construct the IAR map, we first compute the expected number of animal crossings through each of these grid cells $\left(A C_{k}\right.$, where $\left.k \in[1, \ldots, M]\right)$ at time $t=0$. To do this, we determine the number of animals that migrate from each cell to every other cell $\left(T P_{a \rightarrow b}\right)$ using the method defined in Section 2.2. This calculation is performed only once, therefore a finer resolution can be used than the one employed for simulating the population dynamics in Section 2.2. For each of these transitions, the cells traversed are determined by drawing a straight line joining the originating cell $(a)$ to the destination cell $(b)$. The values of $A C_{k}$ for the traversed cells are updated by adding $T P_{a \rightarrow b} \times \kappa_{a}$ to the prevailing value for $A C_{k}$.

Finally, once the matrix AC has been completed, the initial IAR map (IAR) is computed by multiplying every element in AC with the survival probability $(S P)$ due to a single road crossing and dividing by the total population present at time $t=0$. The overall process for computing the IAR grid is summarised in Algorithm 1. 
Main Algorithm Flow

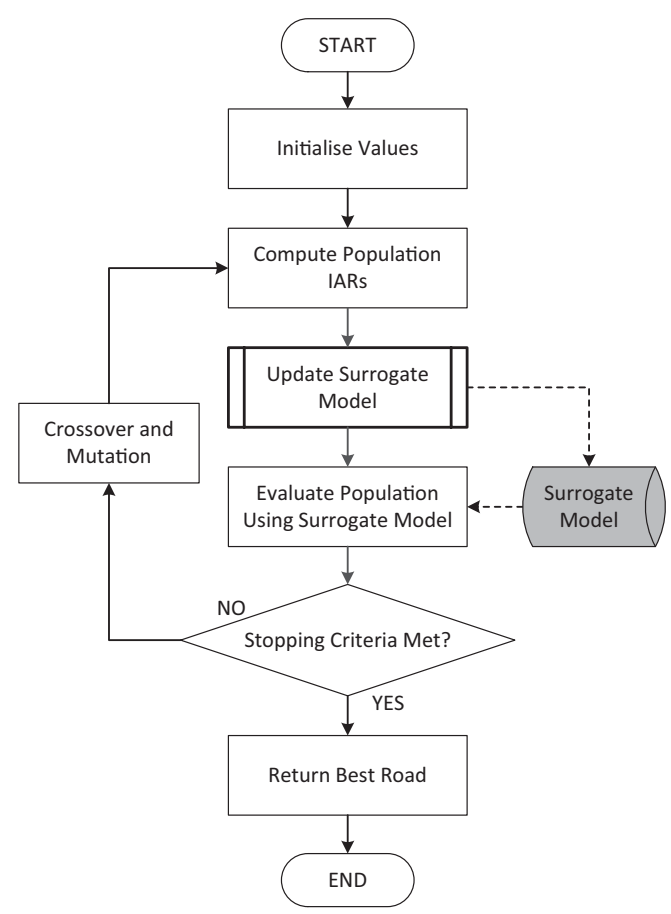

Update Surrogate Model

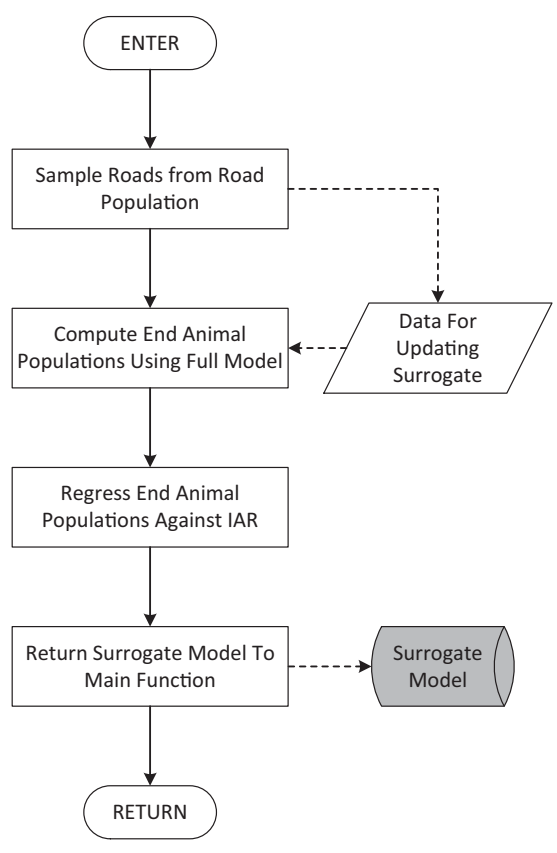

Fig. 3. Program flow for the road design algorithm incorporating animal movement and mortality. The solid lines represent the program flow while the dashed lines indicate information flow.

Algorithm 1. Compute IAR Map.

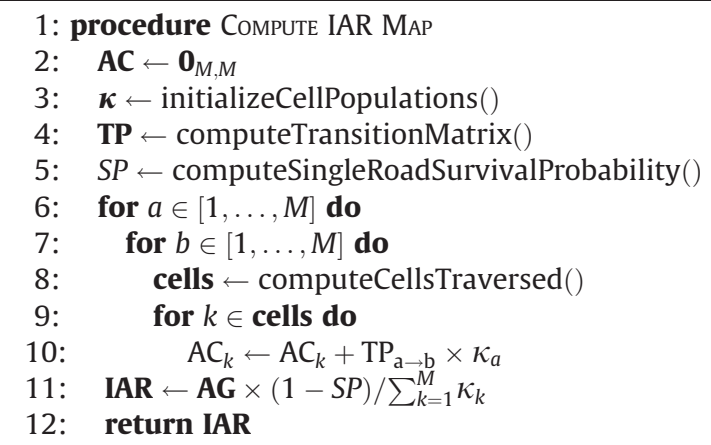

\section{A numerical example}

We explore the application of the new algorithm with a numerical example to observe its effectiveness in selecting a suitable road that satisfies a minimum end population constraint. We simulate terrain that exhibits varied landscape features such as mountains and flat regions that is indicative of the topography of Eastern Australia. The terrain chosen was generated using data from State of Queensland Department of Natural Resources and Mines (2014). This is shown in Fig. 4. We also simulate a habitat map to associate with this terrain as shown in Fig. 5. So as to ensure a non-trivial example, the viable habitat is designated so that the optimal road computed when ignoring species considerations actually passes through much of this habitat.

For this case study, we assume that the prevailing number of animals present in the region at time $t=0$ is at equilibrium. That is, the overall population is equal to the carrying capacity in Eq. (9). For convenience, home ranging behaviour is not included in this example. That is, $\beta=0$.

The numerical example seeks to design a road through the region while ensuring that a surrounding koala population is above a minimum threshold of 900 individuals at the end of the design horizon, $T$. The koala is an iconic arboreal species 


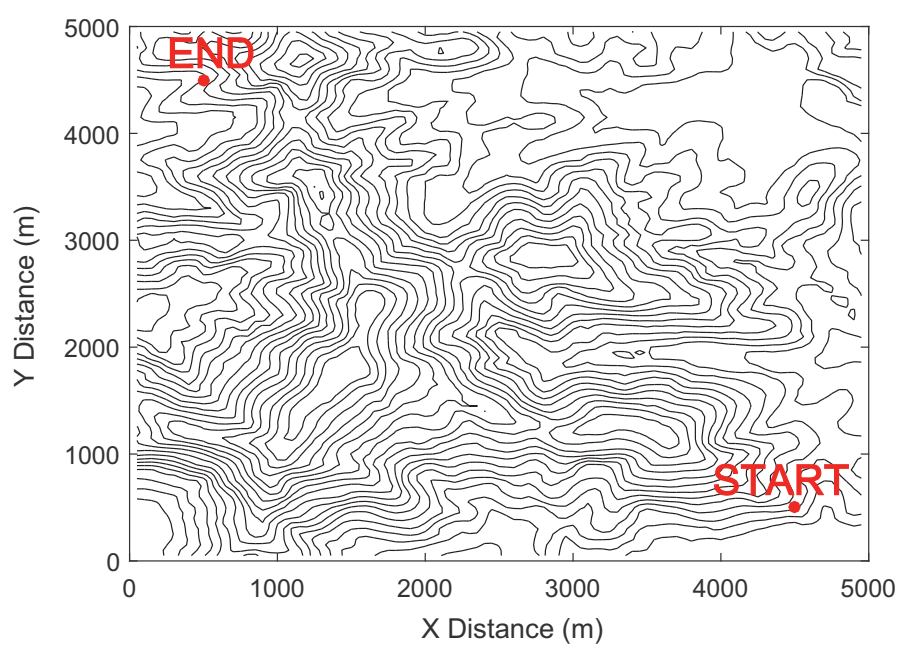

Fig. 4. Topography of the region of interest, showing the start and end points for the road. This is the contour plot for the region introduced in Fig. 1.

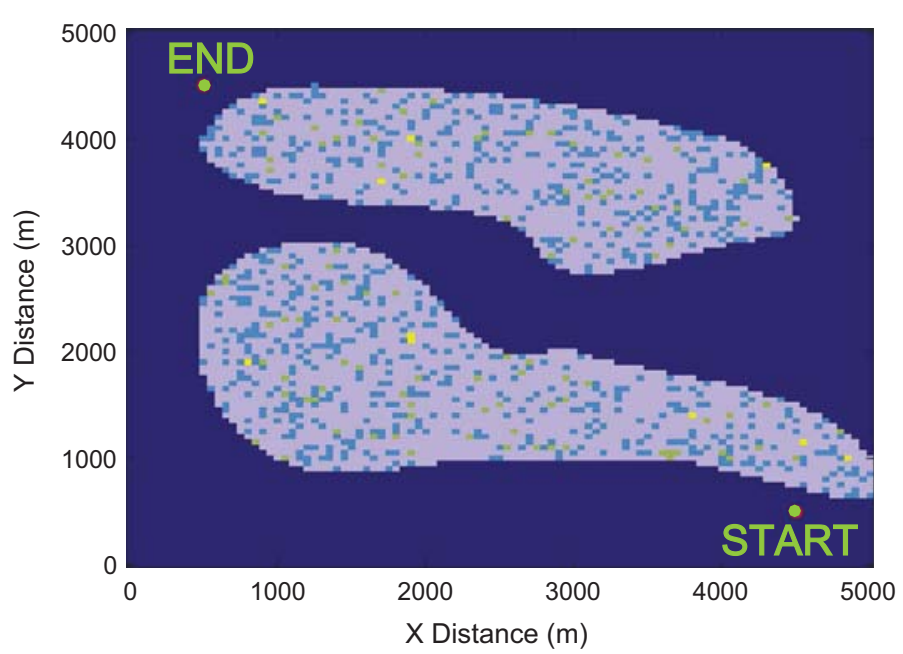

Fig. 5. Locations of viable habitat and initial animal positions in the region. The pale regions indicate viable habitat. These were used to generate the initial animal positions shown by the scattered points.

native to eastern and south-eastern Australia that feeds on specific tree species (Hindell et al., 1985; Phillips et al., 2000). Importantly, a key threat to the species is being struck and killed by vehicles, which typically results from koalas moving across roads when transitioning from one tree to another (Dique et al., 2003b).

The study region is shown in Fig. 4 and is highly irregular, with many hills and valleys. Therefore, the solution space of the road design problem will likely contain many local optima (Jong and Schonfeld, 2003). The road to be designed is a regional highway whose end points are located at $(4500,500,402)$ and $(500,4500,463)$ within the study region. The design data for this problem, such as maximum gradient and desired travel speed are provided in Table 1. Values for the majority of these design parameters are the same as in Jong and Schonfeld (1999), indexed and converted to 2015 Australian Dollars. In addition, earthwork cost values are the same as in Chew et al. (1989), indexed and converted to 2015 Australian Dollars. Finally, the peak time traffic flow rate is set at 1000 vehicles per hour and kept constant for the entire design horizon of $T=50$ years. This peak traffic flow rate is used to compute the traffic throughout the day according to the rule in Jong and Schonfeld (1999) and AASHTO (2011). This flow rate is typical for major regional roads in Australia (State of Queensland Department of Transport and Main Roads, 2015).

The reason for the long horizon is twofold: Firstly, noticeable effects of roads on animal populations require longer time horizons due to gestation periods and for communities to change over time. It becomes hard to assess sustainability of the nearby animal populations if a short horizon is chosen as the population is not given time to possibly reach a steady state or approach extinction. Studies such as (Grimm and Wissel, 2004), estimate mean times to extinction are typically over 100 years. Other ecological papers consulted in this research further illustrate the importance of longer time horizons 
Table 1

Model parameters for the road design model grouped by category. All monetary values are in 2015 Australian dollars (AUD).

\begin{tabular}{ll}
\hline Design Parameters & \\
Maximum road grade & $5^{\circ}$ \\
Design velocity & $80 \mathrm{~km} / \mathrm{h}$ \\
Road width & $20 \mathrm{~m}$ \\
Number of intersection points & 15 \\
Side slope, cut & $45^{\circ}$ \\
Side slope, fill & $30^{\circ}$ \\
Traffic Parameters & \\
Average hourly traffic flow rate & 1000 vehicles/h \\
Traffic growth rate & $0 \%$ p.a. \\
Petrol price & 1.10 \\
Diesel price & 1.00 \\
Accident cost & 4852 \\
Average vehicle width & $2.0 \mathrm{~m}$ \\
Design period & 50 years \\
Traffic growth rate & $0 \%$ p.a. \\
Discount rate & $5 \%$ p.a. \\
Optimisation Parameters & \\
Number of simulations in mortality model for each road & 2000 \\
GA Population & 600 \\
Mutation Rate & 0.40 \\
\hline
\end{tabular}

(Polak et al., 2014; Rhodes et al., 2014; van Langevelde and Jaarsma, 2009; van Langevelde et al., 2009). Secondly, roads are expensive, long-term structures that provide benefits over horizons of many years. Therefore, they will affect animal populations as well as transport networks for many years to come.

A direct path between the start and end points would initially seem infeasible due to the many hills and valleys encountered, which would incur high earthwork costs. It is anticipated that the optimal road alignment in the absence of ecological considerations would instead follow the contour of the mountains or follow the lower-lying and relatively flatter terrain along the top right of the map.

In addition to the topography, a habitat map is provided to represent either animal habitat or non-habitat at each terrain location. The region is split into a $100 \times 100$ grid, where each grid cell is $50 \mathrm{~m} \times 50 \mathrm{~m}$, and the number of animals present in each grid cell at time $t=0$ is determined. Within the viable habitat region, 1000 animals are randomly scattered as shown in Fig. 5. The ecological input parameters required in the animal movement and mortality model are the same as those provided in Rhodes et al. (2014) and are summarised in Table 2.

For this example, the road is defined by $N=15$ intersection points, whose locations are to be determined by the road design model defined in Section 2.1. In addition, the road must satisfy a minimum conservation benefit of $\rho_{\min }=90 \%$ $\left(K_{\min }=900\right)$ at the end of the design horizon. The conservation benefit is computed using the model defined in Section 2.2. The GA is run 7 times and for each of these runs the results of the lowest-cost road are saved. On a $3.60 \mathrm{GHz}$ quad-core PC with 16 GB of RAM running 4 parallel threads in MATLAB r2016a, each GA run takes approximately 5 h to compute. For each of these runs, the GA is computed for up to 2000 generations or until the fitness of the best road is within $0.1 \%$ of the previous best fitness so far.

The corresponding lowest-cost roads among the 7 runs for each test are shown in Fig. 6. Fig. 6a, c and e highlights the amount that each road encroaches on the viable habitat while Fig. $6 \mathrm{~b}, \mathrm{~d}$ and $\mathrm{f}$ shows the end population in each patch, where lighter regions correspond to higher animal populations. These patches were formed by using the patch-creation process outlined in Section 2.2. The road paths also include horizontal and vertical curves situated at the points of intersection in accordance with AASHTO (2011). The real roads incorporating these curves were used to evaluate the end animal populations.

Table 2

Ecological parameters used in the example.

\begin{tabular}{ll} 
Ecological Parameters & \\
Relative preference for cleared land $\left(\alpha_{\text {clear }}\right)$ & -0.373 \\
Negative exponential scale parameter $(\lambda)$ & $-2.52 \times 10^{-3}$ \\
Population growth rate mean $\left(g_{\mu}\right)$ & $0.5 \%$ p.a. \\
Population growth rate standard deviation $\left(g_{\sigma}\right)$ & $1.4 \%$ p.a. \\
Animal length $(l)$ & $0.7 \mathrm{~m}$ \\
Animal crossing speed $(v)$ & $10,000 \mathrm{~m} / \mathrm{h}$ \\
Penalty per animal below required threshold $\left(C_{\text {per_animal }}\right)$ & AUD $10 \mathrm{M}$ \\
Starting population $\left(K_{0}\right)$ & 1000 \\
End population threshold $\left(K_{\min }\right)$ & 900 \\
\hline
\end{tabular}




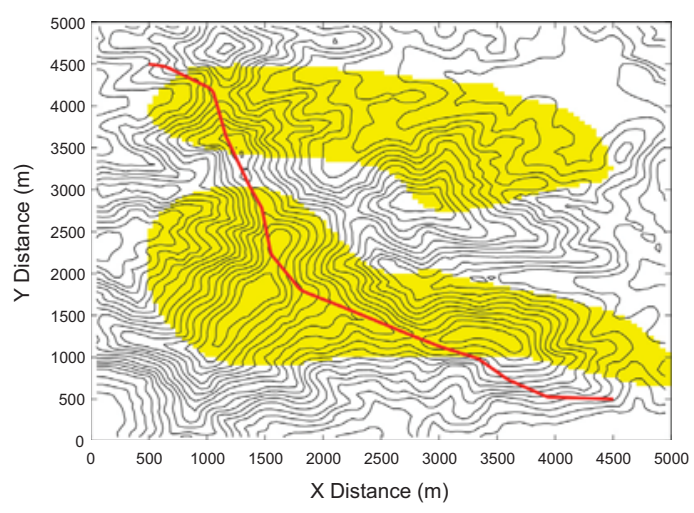

(a) Road path against terrain and habitat - Ignore

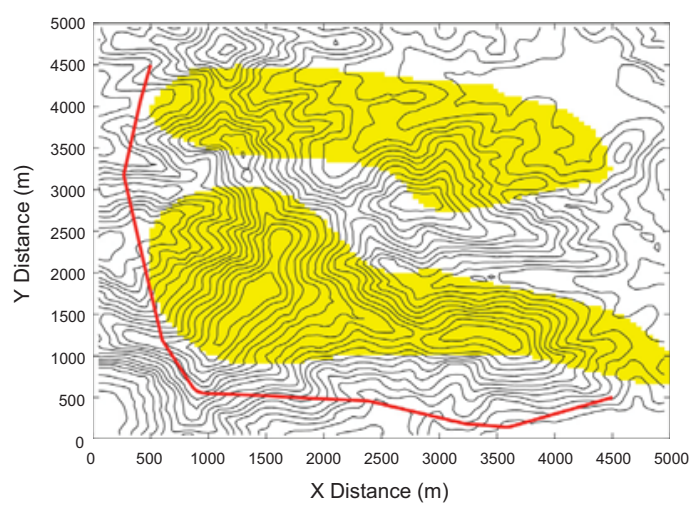

(c) Road path against terrain and habitat - Avoid

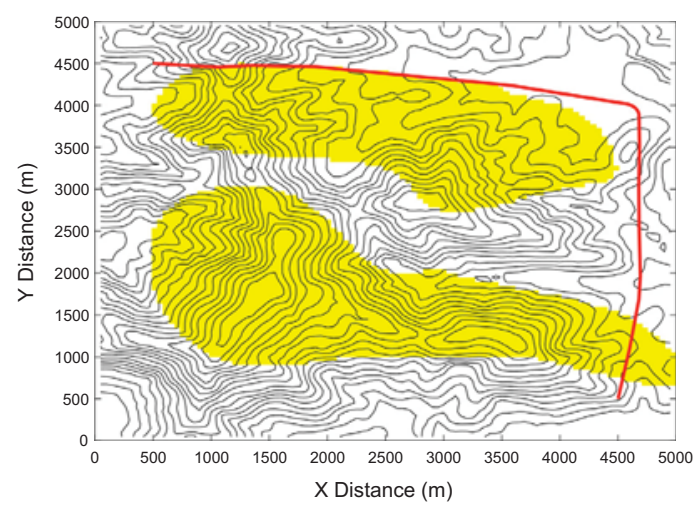

(e) Road path against terrain and habitat - Min

Pop. 900

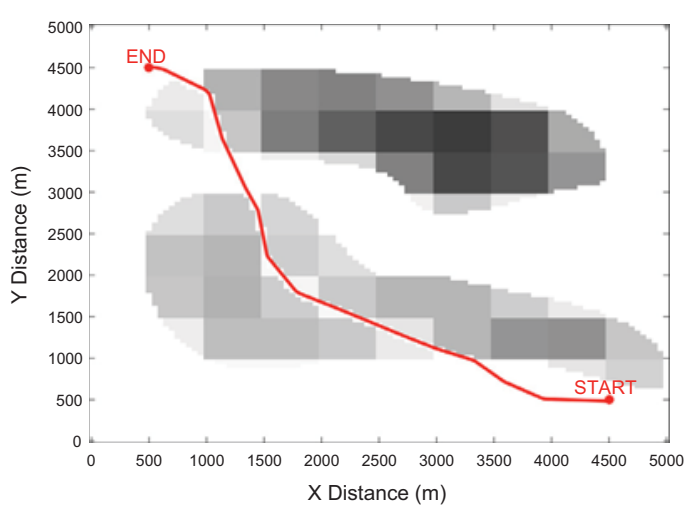

(b) Population map at time $T=50$ years - Ignore

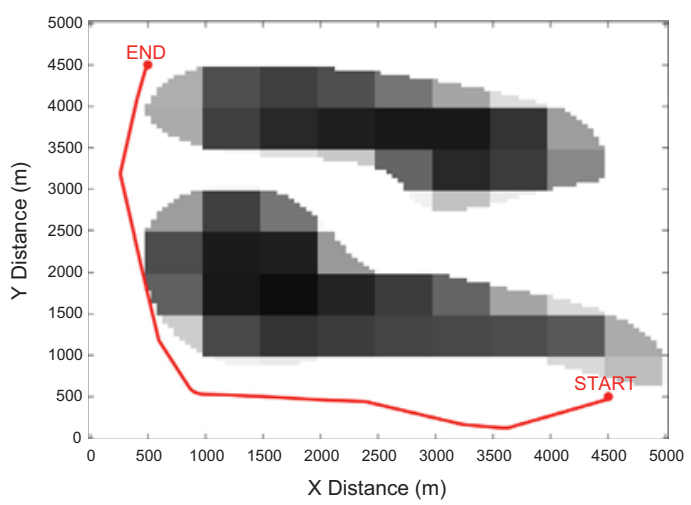

(d) Population map at time $T=50$ years - Avoid

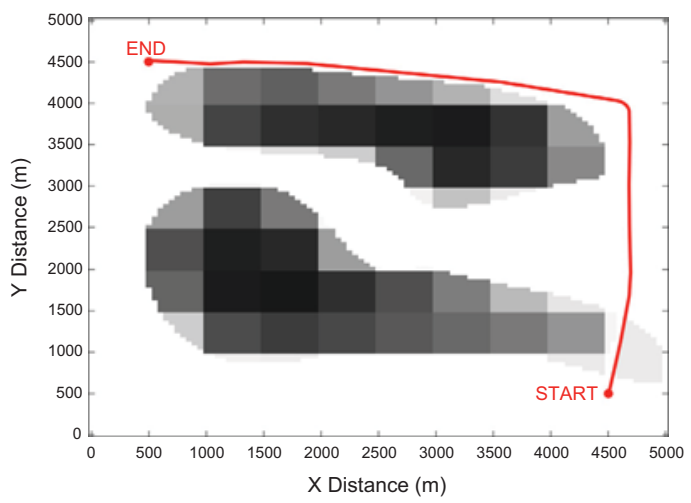

(f) Population map at time $T=50$ years - Min.

Pop. 900

Fig. 6. Paths of the best roads when the animal habitat is ignored (a-b), avoided (c-d) or when a minimum animal population of 900 animals must be satisfied (e-f). Figure b, d and f shows the corresponding end populations in each of the three scenarios, where darker patches correspond to higher populations.

When habitats are ignored, the optimal road follows the ridge of the mountainous region. This path is relatively direct and does not encounter large variations in elevation that would necessitate extensive earthwork. However, this road passes through much of the viable habitat, fragmenting large pockets of animals from each other. This is reflected in a very low conservation benefit of only 58.0\% (580 animals). Conversely, when habitats must be avoided entirely, the optimal road passes through the more undulating terrain of the bottom-left portion of the map. This road encounters many elevation changes along the way that result in higher earthwork and journey costs. The corresponding conservation benefit however, is $100 \%$, indicating that this road has no adverse impacts on the animal population. 
Compared with these two runs, our model explicitly computes the estimated end animal population. The best road produced using this model follows relatively flat terrain along the top and right portions of the map. However, this road avoids more of the viable habitat so as to reduce animal mortality. To achieve this reduced damage, this road traverses terrain that results in greater expenses. However, as the results in Table 3 show, this road is not as expensive as the road produced by the model that avoids animal habitats completely. For this particular case study, the lowest cost road produced by the model incorporating animal movement is $10-15 \%$ more expensive than the lowest cost road when animal habitats are ignored. In contrast, the lowest cost road produced when viable habitat must be avoided is more than twice as expensive. Additionally, the road produced when accounting for animal movement and mortality satisfies the set threshold conservation benefit of $90 \%$. This exceeds the conservation benefit of the lowest cost road when ecological effects are ignored by a considerable margin.

It should be noted that the results produced by the algorithm are highly dependent on the terrain, the location of the viable habitat and the underlying ecological parameters. For example, if the best road found when animal habitats are ignored avoids viable habitat, then the best roads produced under the three cases presented here should be almost the same (allowing for the stochastic nature of the GA). However, if the lowest-cost road produced when animal habitats are ignored traverses significant amounts of viable habitat, as is the case in this example, then this model is highly beneficial.

The example used here contains a number of approximations regarding input data. Road design approximations include but are not limited to: approximate ground surface measurements, course underground material estimation, choice of roadbuilding machinery and equipment, acquisition costs, clearance costs, the composition of traffic over time and future vehicle costs. When implementing road design techniques in practice, accurate and comprehensive input data for all of these inputs must be obtained otherwise the benefits of accounting for animal movement and mortality may be overshadowed by errors in the input data. The road design algorithm used in this paper is sufficiently-developed to incorporate such practical details, as has been explored in previous studies (Jong et al., 2000; Kang et al., 2011, 2012; Kim et al., 2005; Yang et al., 2014).

The ecological model also contains some approximations that should be noted. Firstly, the initial populations are aggregated into habitat patches, as explained in Section 2.2. A large enough number should be chosen to allow capturing the spatial dynamics of the population but must not be too large so as to reduce problem tractability. Secondly, the animal movement model currently does not include other behavioural and environmental characteristics of the species such as road avoidance or threats from other sources. These may be added in future work by using additional models such as those of Friedrich (2015). Finally, the species map is initialised using a uniform distribution over habitable regions. As the example used is not based on a real region, this was deemed appropriate. In reality, species have a certain amount of population dispersal in habitat areas (Dique et al., 2003a; Polak et al., 2014) that will affect the initial positions of the animals. Although this would alter the input population data, it does not affect the validity of the approach investigated in this paper.

\subsection{Evolution of the surrogate model}

The final surrogate model at completion of one of the GA runs is shown in Fig. 7. This plot shows a distinct relationship between initial IAR and the final population achieved. As the GA progresses, the population of roads will contain solutions that are more likely to be closer to the global optimum than in previous generations. This causes the algorithm to focus its search in narrower corridors in the map, where roads with similar IAR values have very similar end populations. This contrasts with earlier stages in the GA where roads with similar IAR values may have vastly different routes and potentially larger differences in their expected final animal populations. Therefore, as the algorithm progresses, the roads in the GA population tend to more closely reflect the true relationship between IAR and final animal population in the vicinity of the lowest-cost road. This reduces the overall variance of the regression plot over time, as reflected in Fig. 8, which shows that the standard error of the surrogate model quickly diminishes to within $\sim 5 \%$ after a few generations. If the algorithm finds a search space that is more viable than the current best search space, then the error of the surrogate model may increase temporarily before decreasing once again as the algorithm searches this new space more thoroughly.

When computing the surrogate, the model samples roads on which to perform the full runs evenly across generations and randomly within each generation. The reason for the larger number of points within the range 0.01-0.04 in Fig. 7 can be attributed to the fact that the algorithm is identifying many roads with these IAR values in the GA population at each stage and comparatively fewer above and below this range. This is reflected in Fig. 9, where most roads fall within this band. For example, higher IAR values likely correspond to long, winding roads that impede animal movement considerably. However, because of their length and high construction costs, they form a smaller and smaller portion of the population over time. The red points at the bottom of Fig. 9 correspond to the lowest-cost road's IAR at each generation.

Table 3

Details of the lowest cost road computed for each scenario in the case study beginning with an initial animal population of 1000 .

\begin{tabular}{llll}
\hline Minimum Conservation Benefit (\%) & Total Cost (AUD \$M) & IAR (\%) & Achieved Conservation Benefit (\%) \\
\hline$\sim 0$ (Ignore Habitats) & 63.0 & 2.88 & 58.0 \\
90 & 71.2 & 0.63 & 91.6 \\
$\sim 100$ (Avoid Habitats) & 143.3 & 0.00 & 100.0 \\
\hline
\end{tabular}




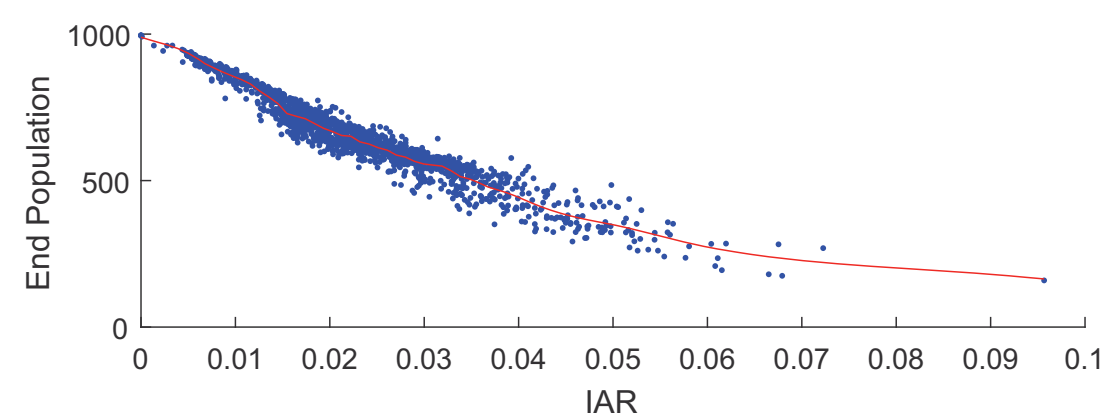

Fig. 7. End population against IAR at the end of a GA run. The blue dots represent data points from full runs that are stored in $\mathcal{D}_{1}$. The red line is the fitted surrogate model that is used to estimate the relationship between the IAR of a road and the species population at the end of the design horizon. (For interpretation of the references to colour in this figure legend, the reader is referred to the web version of this article.)

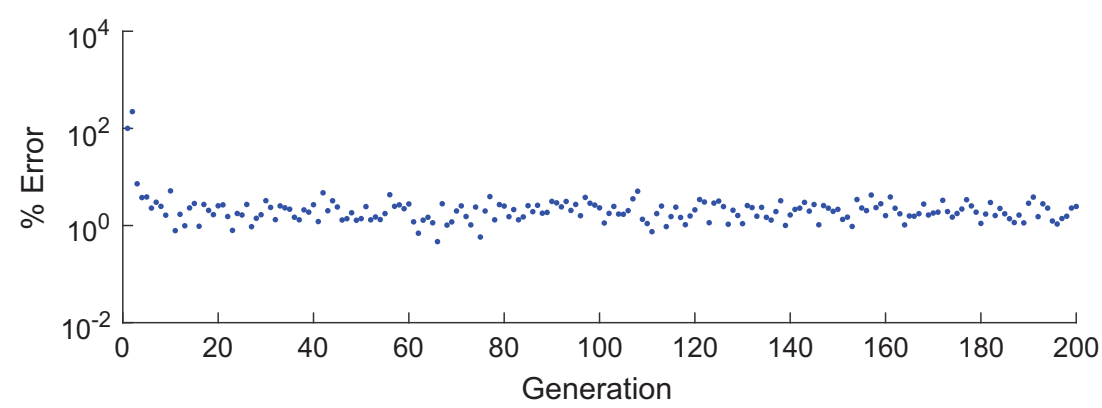

Fig. 8. The standard error of the surrogate function during progression of the GA.

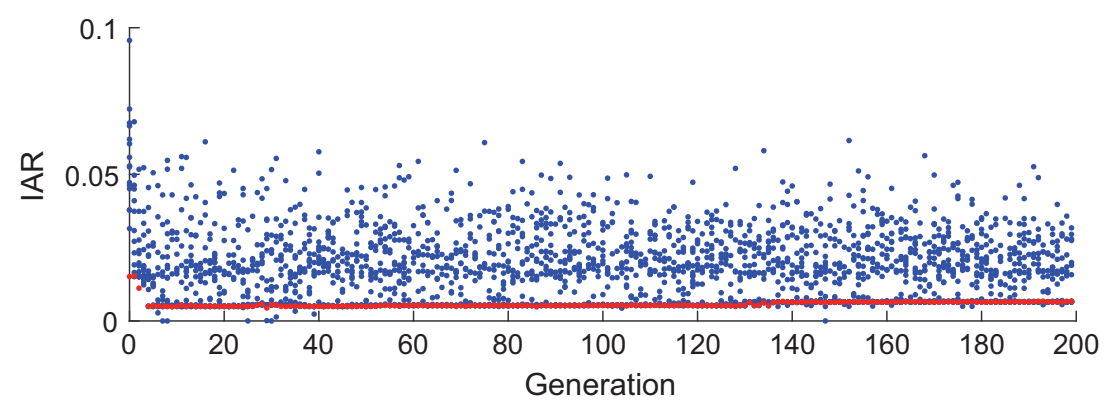

Fig. 9. IARs of sample roads at each generation computed using the full model. The red points correspond to the lowest-cost road in each generation. (For interpretation of the references to colour in this figure legend, the reader is referred to the web version of this article.)

\subsection{Initial Animals at Risk (IAR) map}

The animal movement and mortality model is also used to compute an IAR map (Fig. 10) that provides a visual representation of the contribution of individual map locations to IAR. In this map, the lighter regions reflect areas that, if a road is built through them, will contribute more to IAR. That is, it is comparatively more damaging for the road to pass through these locations. Conversely, the darker regions indicate a smaller contribution to IAR and therefore, less damage.

For the most part, the IAR map of Fig. 10 conforms to the general shape of the inhabited regions shown in Fig. 5. It also shows that more damage is incurred deeper into the habitat regions away from the boundaries. Interestingly, the map also shows that some damage will occur if the road passes between the two patches near coordinate position $(1500,3250)$. This is not surprising as it is possible for animals to migrate between the two patches in this area due to their close proximity. In addition, the map also reveals regions of viable habitat that are not as damaging to travel through near grid locations (2350, $4000),(3200,1500)$ and $(5000,1000)$. From Fig. $6 e$, it can be seen that the resulting road of our algorithm only passes through these low damage locations, which explains the low IAR of the road produced. On the other hand, current road design algorithms are incapable of capturing and exploiting details such as these. Instead, they assume that all areas of 


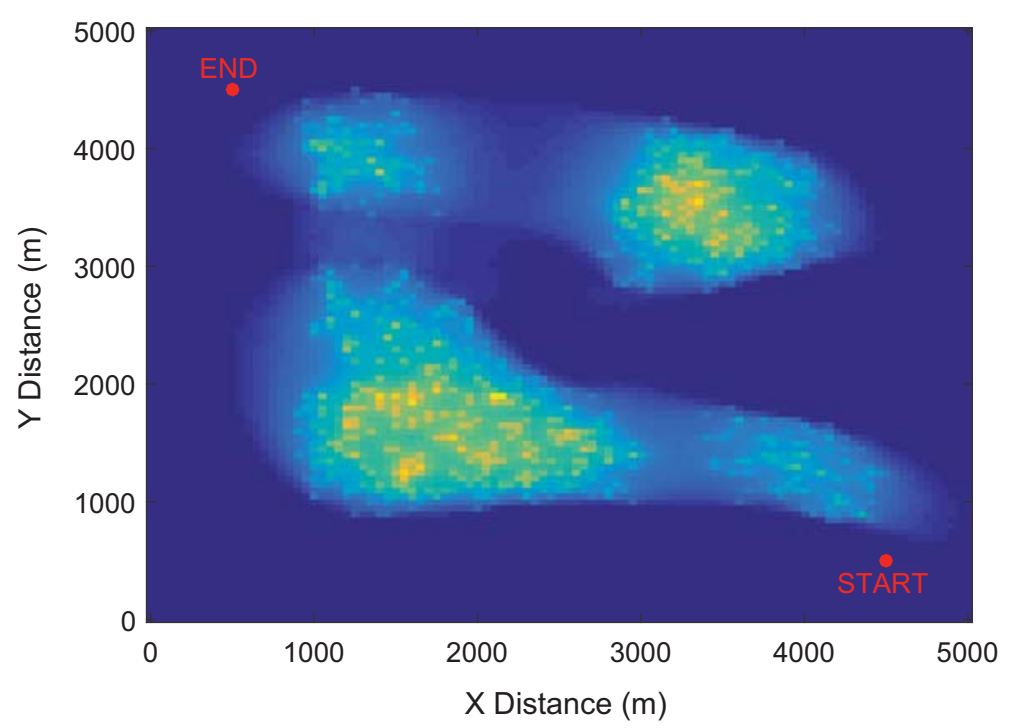

Fig. 10. IAR map for the study region showing the contributions to IAR for each grid location if it is traversed by the road.

the same habitat type incur exactly the same level of damage and that non-habitat would not incur any damage. The IAR map demonstrates that this is not actually the case.

\section{Varying the required conservation benefit and traffic flow rate}

Additional tests are carried out on the example of Section 3 to observe the effect of varying the required conservation benefit and traffic flow rate on the road selected. Five minimum conservation benefits are tested: $50 \%, 60 \%, 70 \%, 80 \%$ and $90 \%$. Additionally, three average traffic flow rates are also tested: 500 vehicles/h, 1000 vehicles/h and 1500 vehicles/h. All other parameters are kept the same as those used for the numerical example in Section 3. The runs are performed in order of increasing required conservation benefit. With each successive increase in required conservation benefit, the lowest-cost road from the previous conservation benefit scenario is provided to the initial GA population of the next run so that the algorithm starts with a low-cost road in the population.

Due to the stochastic nature of the GA road design algorithm used, each of these cases is run 7 times ( 35 runs in total) and the results of the lowest-cost road found are recorded. The total cost, IAR and achieved conservation benefit under each minimum population threshold are provided in Fig. 11. These results show that the algorithm introduced in this paper is capable of meeting the minimum conservation benefit, with the difference between the achieved and threshold conservation benefits decreasing as the minimum required conservation benefit is increased.

Even though the costs of the best roads found for each conservation benefit are similar for a fixed flow rate, their achieved conservation benefits increase in order to satisfy the required conservation benefit. This demonstrates the ability of the algorithm to find low-cost roads that satisfy a specified conservation benefit. On the other hand, the original algorithms that simply ignore or avoid habitat regions cannot measure ecological impacts and therefore have no way of differentiating between roads with higher impacts. They are therefore unable to select roads that meet a minimum population constraint.

As one may expect, the traffic flow rate has a significant effect on the end population of the best road selected. This is seen most clearly by the end population for the road that ignores the habitat zones. In the case with a flow rate of 500 vehicles an hour, the population was consistently maintained above 650 animals by the end horizon. However, this end population decreased as the flow rate increased for population thresholds below 700 animals. For the higher minimum conservation benefits, this forced the algorithm to explore different areas of the map to find feasible roads.

The overall road costs are also higher for larger traffic flow rates. This is to be expected because the operating component of the road costs increases due to higher traffic volumes having higher running costs. Additionally, as the minimum population threshold is increased, the algorithm is more likely to select roads that have a lower impact. For this study region, these correspond to more expensive roads. This is most pronounced for the population threshold of 900 animals, for which the cost of the best road increases the most as the flow rate is increased. As the traffic flow rate has a significant effect on the final road chosen, road designers may be able to use the algorithm together with a suitable rate that satisfies a desired minimum end population that also meets a desired project budget. Alternately, they could devise a set of flow rates with different animal impacts to switch between based on the prevailing animal population.

Furthermore, as the overall road optimisation algorithm is based on a genetic algorithm, no guarantee is provided on its consistency between runs or in finding the global optimum. This is complicated by the presence of multiple local optima and 


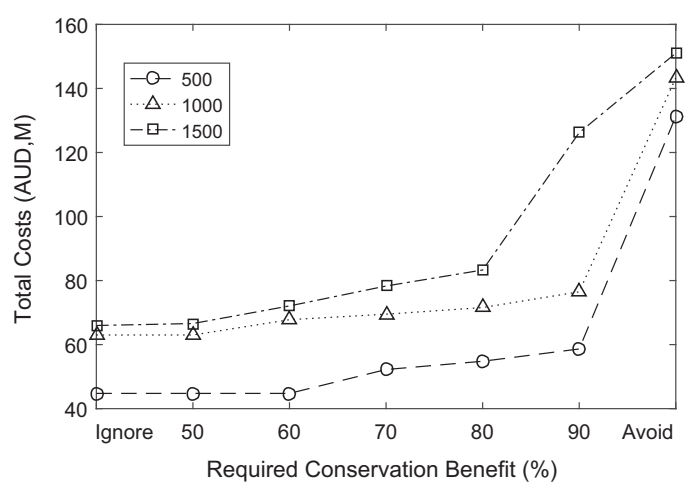

(a) Lowest cost road

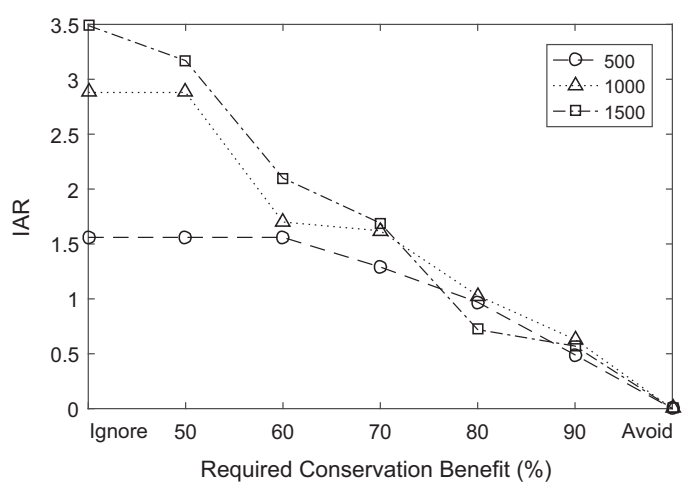

(b) Lowest cost road IAR

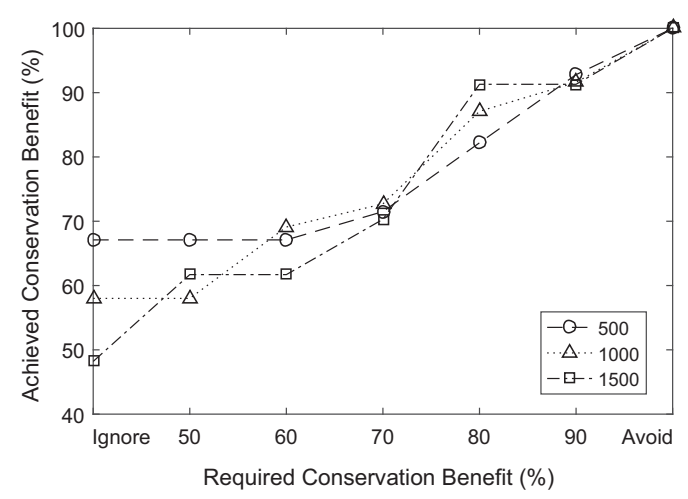

(c) Lowest cost road achieved conservation benefit

Fig. 11. Results of the best runs for each required conservation benefit for three traffic flow rates (500, 1000, 1500 vehicles per hour).

complex terrain encountered in real-world scenarios. In such cases, the optimisation process will require more executions for a user to be confident of finding a suitable low-cost road. This suggests that the solution space becomes more complex with the increase in the constraint, but the difficulty in searching it is a property of the GA and not necessarily an indication of the number of feasible roads available.

Increasing the population size and the number of generations over which the model is run as well as fine tuning the mutation and crossover ratios may improve the value of the lowest-cost road for each required conservation benefit. In addition, computational gains may be achieved by using derivative-free optimisation algorithms with multi-start as implemented in NOMAD and HOPSPACK as suggested by Mondal et al. (2015). Finally, further improvements in computation time may be achieved by using a compiled language and or for example, employing GPUs, distributed computing or multiple core systems.

\section{Conclusion and future work}

This paper developed a framework for designing a road through an ecologically-sensitive area. Unlike existing road design algorithms, the approach presented in this paper explicitly accounts for the expected species mortality due to the road design. It does this by incorporating an empirically-tested ecological model into a road design model based on the genetic algorithm to determine the effects of vehicle collisions on animal mortality. This detailed approach has not been considered until now. To allow computational tractability of the overall road design algorithm incorporating ecological dynamics, two concepts have been introduced. The first of these is Initial Animals at Risk, which reduces the number of predictors of the end population of a road from three times the number of design points $(3 N)$ to a single variable called IAR. IAR has physical meaning, representing the proportion of animals expected to perish in the first time period due to a particular road design. The second concept is the surrogate model that is updated as the GA progresses. The surrogate model represents the functional relationship between the IAR of a road and the expected end population at the end of the design horizon. By using this model, the GA only needs to compute the full animal movement and mortality for a small number of roads, drastically improving computational tractability.

Numerical tests show that the algorithm is able to learn the surrogate model effectively, owing to the exploratory behaviour of the GA. As the algorithm focuses its search in more promising regions of the solution space with each successive 
generation, the error between full model runs and the value estimated by the surrogate function quickly diminishes. Running the full model on the final roads produced by each GA run shows that the algorithm is consistently capable of finding a lowcost road that satisfies the end population constraint. Importantly, when compared with the roads produced by the existing algorithm, the model proposed in this paper is able to improve the expected end population of the road without incurring unnecessary costs caused by having to avoid habitat regions altogether. Therefore, the algorithm is an important design tool that allows road designers to evaluate a road's ecological impact during preliminary design rather than after. This will ultimately lead to more sustainable low-cost road designs.

The work presented in this paper is an important first step in accounting for ecological uncertainty in road design and has much scope to be extended in future research. Such future work might include a sensitivity analysis on the effects of different input ecological factors that may affect the outcome of the final road selected. Notably, this could consider more realistic initial population distributions based on real world data that take into account species dispersal. Other avenues include accounting for additional migration behaviours in the presence of roads or when multiple species must be assessed.

This paper also treated traffic flow as static over the design life, whereas in reality, traffic can be controlled over time. Therefore, future work may also investigate the effects of adding switching flexibility to a proposed design to alter traffic based on prevailing animal population levels. Finally, there is a possibility of including other mitigation techniques such as the inclusion of optimally-located wildlife bridges in the road design process.

\section{Acknowledgements}

The authors would like to thank Mukesh Soni, Khalid Abdulla and the three anonymous reviewers for their helpful comments, which have greatly improved the quality of the manuscript.

This research was funded through an Australian Government Research Training Program Scholarship.

\section{References}

AASHTO, 2011. A Policy on Geometric Design of Highways and Streets. sixth ed., American Association of State Highway and Transportation Officials, Washington, DC

Abramson, M., Audet, C., Couture, G., Dennis Jr., J., Le Digabel, S., Tribes, C., 2016. The NOMAD Project. <http://ww.gerad.ca/nomad>.

Alexander, S.M., 2008. Snow-tracking and GIS: using multiple species-environment models to determine optimal wildlife crossing sites and evaluate highway mitigation plans on the Trans-Canada Highway. Can. Geogr. 52, 169-187. http://dx.doi.org/10.1111/j.1541-0064.2008.00207.x.

Alexander, S.M., Waters, N.M., 2000. The effects of highway transportation corridors on wildlife: a case study of Banff National Park. Transp. Res. Part C, 307320. http://dx.doi.org/10.1016/S0968-090X(00)00014-0.

Aruga, K., 2005. Tabu search optimization of horizontal and vertical alignments of forest roads. J. Forest Res. 10, 275-284. http://dx.doi.org/10.1007/s10310004-0136-5.

Audet, C., Dennis, J., 2006. Mesh adaptive direct search algorithms for constrained optimization. SIAM J. Optim. 17, 188-217. http://dx.doi.org/10.1137/ 040603371.

Audet, C., Le Digabel, S., Tribes, C., 2009. NOMAD User Guide. Technical Report G-2009-37. Technical Report.

Casal, G., Santamarina, D., Vázquez-Méndez, M.E., 2017. Optimization of horizontal alignment geometry in road design and reconstruction. Transp. Res. Part C 74, 261-274. http://dx.doi.org/10.1016/j.trc.2016.11.019.

Chew, E., Goh, C., Fwa, T., 1989. Simultaneous optimization of horizontal and vertical alignments for highways. Transp. Res. Part B 22, 315-329. http://dx. doi.org/10.1016/0191-2615(89)90008-8.

Cruz, J.M., Wakolbinger, T., 2008. Multiperiod effects of corporate social responsibility on supply chain networks, transaction costs, emissions, and risk. Int. J. Prod. Econ. 116, 61-74. http://dx.doi.org/10.1016/j.ijpe.2008.07.011.

Davey, N., Dunstall, S., Halgamuge, S., 2016. The value of flexible road designs through ecologically sensitive areas. In: Lecture Notes in Management and Industrial Engineering, Canberra, Australia, in press

De Smith, M.J., 2006. Determination of gradient and curvature constrained optimal paths. Comput.-Aided Civil Infrastruct. Eng. 21, 24-38. http://dx.doi.org/ $10.1111 / \mathrm{j} .1467-8667.2005 .00414 \mathrm{x}$.

Dique, D.S., Thompson, J., Preece, H.J., de Villiers, D.L., Carrick, F.N., 2003a. Dispersal patterns in a regional koala population in south-east queensland. Wildlife Res. 30, 281-290.

Dique, D.S., Thompson, J., Preece, H.J., Penfold, G.C., de Villiers, D.L., Leslie, R.S., 2003b. Koala mortality on roads in south-east Queensland: the koala speedzone trial. Wildlife Res. 30, 419-426.

Fahrig, L., 2001. How much habitat is enough? Biol. Conserv. 100, 65-74. http://dx.doi.org/10.1016/S0006-3207(00)00208-1.

Fahrig, L., 2003. Effects of habitat fragmentation on biodiversity. Annu. Rev. Ecol. Evol. Syst., 487-515

Fahrig, L., Rytwinski, T., 2009. Effects of roads on animal abundance: an empirical review and synthesis. Ecol. Soc. 14, 21-41 <http://www. ecologyandsociety.org/vol14/iss1/art21/>.

Forman, R.T.T., Alexander, L.E., 1998. Roads and their major ecological effects. Annu. Rev. Ecol. Syst. 29, 207-231. http://dx.doi.org/10.1146/annurev. ecolsys.29.1.207.

Forrester, A., Sobester, A., Keane, A., 2008. Engineering Design via Surrogate Modelling: A Practical Guide. John Wiley \& Sons, England.

Frair, J.L., Merrill, E.H., Beyer, H.L., Morales, J.M., 2008. Thresholds in landscape connectivity and mortality risks in response to growing road networks. J. Appl. Ecol. 45, 1504-1513. http://dx.doi.org/10.1111/j.1365-2664.2008.01526.x.

Frankham, R., 1995. A threshold and extinction: inbreeding. Conserv. Biol. 9, 792-799.

Frankham, R., 1996. Relationship of genetic variation to population size in wildlife. Conserv. Biol. 10, 1500-1508. http://dx.doi.org/10.1046/j.15231739.1996.10061500.x.

Friedrich, J., 2015. Integrating neglected ecological impacts of road transport into corporate management. Ecol. Ind. 54, 197-202. http://dx.doi.org/10.1016 j.ecolind.2015.01.026.

Gorissen, D., Couckuyt, I., Demeester, P., Dhaene, T., Crombecq, K., 2010. A surrogate modeling and adaptive sampling toolbox for computer based design. J Mach. Learn. Res. 11, 2051-2055 <http://dl.acm.org/citation.cfm?id=1859919>.

Grimm, V., Wissel, C., 2004. The intrinsic mean time to extinction: a unifying approach to analysing persistence and viability of populations. Oikos $105,501-$ $511<$ http://www.jstor.org/stable/3548286>.

Hare, W., Hossain, S., Lucet, Y., Rahman, F., 2014. Models and strategies for efficiently determining an optimal vertical alignment of roads. Comput. Oper. Res. 44, 161-173. http://dx.doi.org/10.1016/j.cor.2013.11.005.

Hastie, T., Tibshirani, R., Friedman, J., 2009. The Elements of Statistical Learning. Springer, Berlin. http://dx.doi.org/10.1007/978-0-387-84858-7. 
Hindell, M., Handasyde, K., Lee, A., 1985. Tree species selection by free-ranging koala populations in Victoria. Wildlife Res. 12, 137-144.

Holderegger, R., Di Giulio, M., 2010. The genetic effects of roads: a review of empirical evidence. Basic Appl. Ecol. 11, 522-531.

Jaarsma, C.F., 1997. Approaches for the planning of rural road networks according to sustainable land use planning. Landscape Urban Plann. 39, 47-54. http://dx.doi.org/10.1016/S0169-2046(97)00067-4.

Jaeger, J.A.G., Bowman, J., Brennan, J., Fahrig, L., Bert, D., Bouchard, J., Charbonneau, N., Frank, K., Gruber, B., Von Toschanowitz, K.T., 2005. Predicting when animal populations are at risk from roads: an interactive model of road avoidance behavior. Ecol. Model., 329-348 http://dx.doi.org/10.1016/j. ecolmodel.2004.12.015.

Jha, M.K., Schonfeld, P., Jong, J.C., 2006. Intelligent Road Design, vol. 19. WIT Press.

Jin, Y., 2005. A comprehensive survey of fitness approximation in evolutionary computation. Soft. Comput. 9, 3-12. http://dx.doi.org/10.1007/s00500-0030328-5.

Jin, Y., 2011. Surrogate-assisted evolutionary computation: recent advances and future challenges. Swarm Evol. Comput. 1, 61-70. http://dx.doi.org/ 10.1016/j.swevo.2011.05.001.

Jong, J.C., Jha, M.K., Schonfeld, P., 2000. Preliminary highway design with genetic algorithms and geographic information systems. Comput.-Aided Civil Infrastruct. Eng. 15, 261-271. http://dx.doi.org/10.1111/0885-9507.00190.

Jong, J.C., Schonfeld, P., 1999. Cost functions for optimizing highway alignments. Transp. Res. Rec.: J. Transp. Res. Board 1659, 58-67. http://dx.doi.org/ $10.3141 / 1659-08$.

Jong, J.C., Schonfeld, P., 2003. An evolutionary model for simultaneously optimizing three-dimensional highway alignments. Transp. Res. Part B 37, 107-128. http://dx.doi.org/10.1016/S0191-2615(01)00047-9.

Kang, M.W., Jha, M.K., Schonfeld, P., 2012. Applicability of highway alignment optimization models. Transp. Res. Part C 21, 257-286. http://dx.doi.org/ 10.1016/j.trc.2011.09.006.

Kang, M.W., Schonfeld, P., Yang, N., 2009. Prescreening and repairing in a genetic algorithm for highway alignment optimization. Comput.-Aided Civil Infrastruct. Eng. 24, 109-119. http://dx.doi.org/10.1111/j.1467-8667.2008.00574.x.

Kang, M.W., Yang, N., Schonfeld, P., Jha, M., 2011. Bilevel highway route optimization. Transp. Res. Rec. 2197, 107-117. http://dx.doi.org/10.3141/2197-13.

Kim, E., Jha, M.K., Son, B., 2005. Improving the computational efficiency of highway alignment optimization models through a stepwise genetic algorithms approach. Transp. Res. Part B 39, 339-360. http://dx.doi.org/10.1016/j.trb.2004.06.001.

Lande, R., 1993. Risks of population extinction from demographic and environmental stochasticity and random catastrophes. Am. Nat. 142, 911-927. http:// dx.doi.org/10.2307/2462690.

van Langevelde, F., van Dooremalen, C., Jaarsma, C.F., 2009. Traffic mortality and the role of minor roads. J. Environ. Manage. 90, 660-667. http://dx.doi.org/ 10.1016/j.jenvman.2007.09.003.

van Langevelde, F., Jaarsma, C.F., 2009. Modeling the effect of traffic calming on local animal population persistence. Ecol. Soc., 14

Li, W., Pu, H., Schonfeld, P., Zhang, H., Zheng, X., 2016. Methodology for optimizing constrained 3-dimensional railway alignments in mountainous terrain. Transp. Res. Part C 68, 549-565. http://dx.doi.org/10.1016/j.trc.2016.05.010.

Li, W., Pu, H., Zhao, H., Liu, W., 2013. Approach for optimizing 3d highway alignments based on two-stage dynamic programming. J. Software 8, 2967-2973. http://dx.doi.org/10.4304/jsw.8.11.2967-2973.

Lunney, D., Phillips, S., Callaghan, J., Coburn, D., 1998. Determining the distribution of koala habitat across a shire as a basis for conservation: a case study from Port Stephens, New South Wales. Pac. Conserv. Biol. 4, 186-196.

Maji, A., Jha, M.K., 2012. Comparison of single and multi-objective highway alignment optimization algorithms. Adv. Transp. Stud. 82, 5-16. http://dx.doi. org/10.4399/97888548486721.

Menge, B.A., Sutherland, J.P., 1976. Species diversity gradients: synthesis of the roles of predation, competition, and temporal heterogeneity. Am. Nat., 351369

Mishra, S., Kang, M.W., Jha, M.K., 2014. Empirical model with environmental considerations in highway alignment optimization. J. Infrastruct. Syst. 20. http://dx.doi.org/10.1061/(ASCE)IS.1943-555X.0000194, p. 04014017.

Mondal, S., Lucet, Y., Hare, W., 2015. Optimizing horizontal alignment of roads in a specified corridor. Comput. Oper. Res. 64, 130-138. http://dx.doi.org/ 10.1016/j.cor.2015.05.018.

Phillips, S., Callaghan, J., Thompson, V., 2000. The tree species preferences of koalas (Phascolarctos cinereus) inhabiting forest and woodland communities on quaternary deposits in the Port Stephens area, New South Wales. Wildlife Res. 27, 1-10.

Plantenga, T., 2009. HOPSPACK 2.0 user manual. Technical report SAND2009-6265.

Polak, T., Rhodes, J.R., Jones, D., Possingham, H.P., 2014. Optimal planning for mitigating the impacts of roads on wildlife. J. Appl. Ecol. 51, 726-734. http://dx. doi.org/10.1111/1365-2664.12243.

Prno, J., Slocombe, S.D., 2012. Exploring the origins of 'social license to operate' in the mining sector: perspectives from governance and sustainability theories. Resour. Policy 37, 346-357. http://dx.doi.org/10.1016/j.resourpol.2012.04.002.

Rhodes, J.R., Lunney, D., Callaghan, J., McAlpine, C.A., 2014. A few large roads or many small ones? how to accommodate growth in vehicle numbers to minimise impacts on wildlife. PLoS One, 9. http://dx.doi.org/10.1371/journal.pone.0091093.

Rhodes, J.R., Mcalpine, C.A., Lunney, D., Possingham, H.P., 2005. A spatially explicit habitat selection model incorporating home range behavior. Ecology 86, 1199-1205. http://dx.doi.org/10.1890/04-0912.

Shafahi, Y., Bagherian, M., 2013. A customized particle swarm method to solve highway alignment optimization problem. Comput.-Aided Civil Infrastruct. Eng. 28, 52-67. http://dx.doi.org/10.1111/j.1467-8667.2012.00769.x.

State of Queensland Department of Natural Resources and Mines, 2014. Contours - 5 metre - Central Queensland. <http://qldspatial.information.qld.gov. au/catalogue/custom/search.page?q=\%22Contours-5metre-CentralQueensland\%22> (accessed 3-January-2016).

State of Queensland Department of Natural Resources and Mines, 2016. Vegetation management - regulated vegetation management map - version 1.28. <http://qldspatial.information.qld.gov.au/catalogue/custom/detail.page?fid=536317E8-01C6-4845-86A6-43301B24C9F9> (accessed 28-April-2016).

State of Queensland Department of Transport and Main Roads, 2015. 2014 traffic census data. <https://data.qld.gov.au/dataset/traffic-census-for-thequeensland-state-declared-road-network/resource/26a60055-acf7-409d-8891-593b991c2ff4> (accessed 28-April-2016).

Yang, N., Kang, M.W., Schonfeld, P., Jha, M.K., 2014. Multi-objective highway alignment optimization incorporating preference information. Transp. Res. Part C 40, 36-48. http://dx.doi.org/10.1016/j.trc.2013.12.010. 